\title{
MULTIMODALITY IMAGING OF OSTEOPETROSIS
}

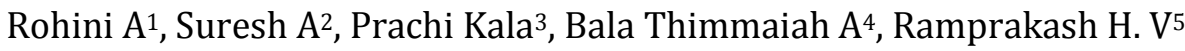

\section{HOW TO CITE THIS ARTICLE:}

Rohini A, Suresh A, Prachi Kala, Bala Thimmaiah A, Ramprakash H. V. "Multimodality Imaging of Osteopetrosis". Journal of Evolution of Medical and Dental Sciences 2014; Vol. 3, Issue 14, April 07; Page: 3721-3726, DOI: $10.14260 /$ jemds/2014/2350

ABSTRACT: Osteopetrosis (OP) is a descriptive term that refers to a group of rare, heritable disorders of the skeleton. This condition varies greatly in their presentation and severity, ranging from neonatal onset with life-threatening complications (classic or "malignant" ARO), to the incidental finding on radiographs (ADO), due to carbonic anhydrase deficiency and an intermediate (IOP) a more uncommon form of the disease. However, this variant tends to present later in childhood. Plain radiography and sectional imaging in particular magnetic resonance imaging (MRI) plays important role in the diagnosis of the osteopetrosis and its subtypes. To demonstrate IOP radiological features, role of different imaging modalities and the importance of imaging in diagnosis especially the role of MRI in diagnosing the type of inheritance in patients of osteopetrosis. We present a case of 7 year old boy of osteopetrosis intermediate type of inheritance, diagnosed by plain radiography with clinical differential diagnosis of Thalassemia major. Plain radiography is the modality of choice in diagnosing the disease. MRI further helps to identify the variants of the osteopetrosis. This case emphasizes the importance of imaging in the diagnosis of the osteopetrosis. KEYWORDS: Osteopetrosis, MRI, variants of OP, plain radiography, Computed Tomography.

INTRODUCTION: Osteopetrosis also known as Albers- Schönberg disease or "marble bone disease" is a descriptive term that refers to a group of rare, heritable disorders of the skeleton in which there is diffuse increase in thickness of the skeleton. The Incidence of most frequent patterns among is autosomal recessive osteopetrosis (ARO) 1 in 250, 000 births, and autosomal dominant osteopetrosis (ADO) 1 in 20, 000 births.1,2 Osteopetrotic conditions vary greatly in their presentation and severity, ranging from neonatal onset with life-threatening complications (classic or "malignant" ARO), to the incidental finding of osteopetrosis on radiographs (ADO)and mild form of autosomal recessive osteopetrosis (mild ARO/intermediate OP) a more uncommon form of the disease. However, this variant tends to present later in childhood than the more malignant form.

Typically, these patients have short stature and experience some of the more aggressive features of malignant osteopetrosis. Frequently IOP is distinguished from the malignant form only when a milder clinical course evolves with age.1-5 Plain radiography and sectional imaging in particular magnetic resonance imaging (MRI) plays important role in the diagnosis of the osteopetrosis and its subtypes.6, 7,8

CASE REPORT: A seven year old male child from a family of lower socio-economic group with a history of abdominal distension and fever since three months and a clinical diagnosis of thalassemia major referred for further investigation to the department of Radiology of Vydehi hospital.

The child was a term baby born at home by normal vaginal delivery. The birth weight was 2.5 $\mathrm{kg}$. Mile stones, social behavior and development history was normal. There is history of recurrent attacks of fever during first three years. Later he started developing abdominal distension, anemia, 
jaundice, weakness, pica and loss of appetite. Child had history of blood transfusions initially once in three months which later increased in frequency to present status, 3-4 times per month. The symptoms used to improve after every transfusion. No history of consanguineous marriage and no significant pre and post natal history. The family history and antenatal history were unremarkable. The younger sibling is 24 months old and is healthy.

At admission the weight of the child was $14 \mathrm{~kg}$ (grade III PEM). Head circumference was $49 \mathrm{~cm}$ and height was $98 \mathrm{~cm}$ (expected $120 \mathrm{~cm}$ ). On physical examination he had anemia, abdominal distension, non-tender hepatomegaly $(11 \mathrm{~cm}$ below the right coastal margin) and nontender splenomegaly ( $20.5 \mathrm{~cm}$ below the left coastal margin). There were bilateral enlarged cervical, axillary and inguinal lymph nodes. CVS, CNS and respiratory system examinations were unremarkable. Ophthalmic examination showed normal binocular vision.

Blood Investigations revealed, Hb 5gm. \%, RBC $2.5 \times 10^{6} / \mu \mathrm{L}$. Peripheral blood smear revealed severe dimorphic anemia with thrombocytopenia. Blood sugar, liver function tests, urea, creatinine, sodium and potassium were within normal limits. Serum calcium was $8 \mathrm{mg} \%$. Blood culture was sterile. HIV spot test was negative. Attempts at bone marrow aspiration yielded only scanty marrow. The cervical lymph nodes aspiration cytology revealed nonspecific lymphadenitis.

Radiographs of limbs, skull and spine revealed generalized increase in bone density. Radiographs of long bones revealed “'bone within bone' appearance with remodeling deformity (Fig 1). Radiograph of spine showed endobone appearance ('rugger jersey') (Fig 2). Radiographs of skull showed sclerosis and thickening of orbital rims (Fig 3) and anterior cranial fossa. Sella turcica was small.

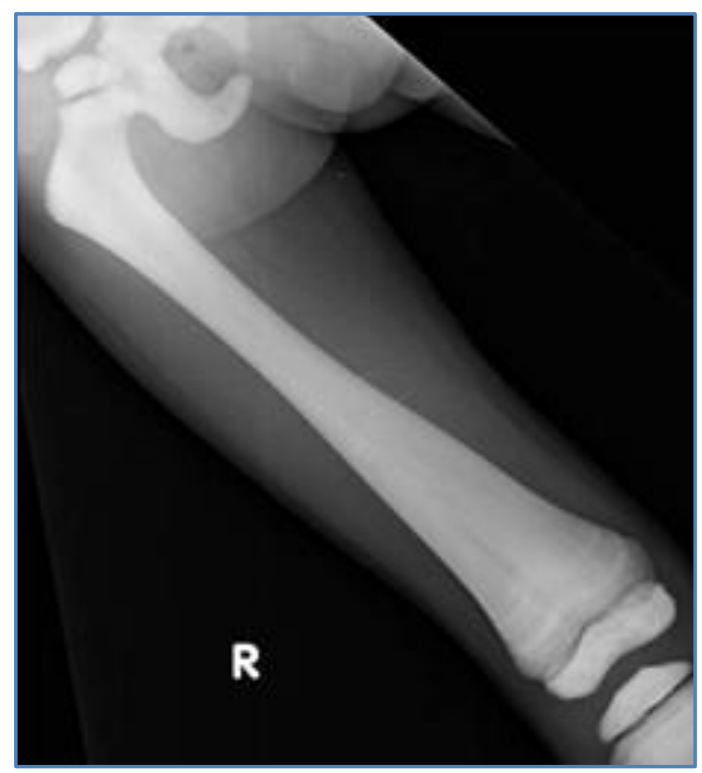

Figure 1: Plain radiogram of right femur: There is generalized sclerosis with bone in bone appearance with remodelling deformity giving appearance of Erlenmeyer flask deformity of distal femur 


\section{CASE REPORT}

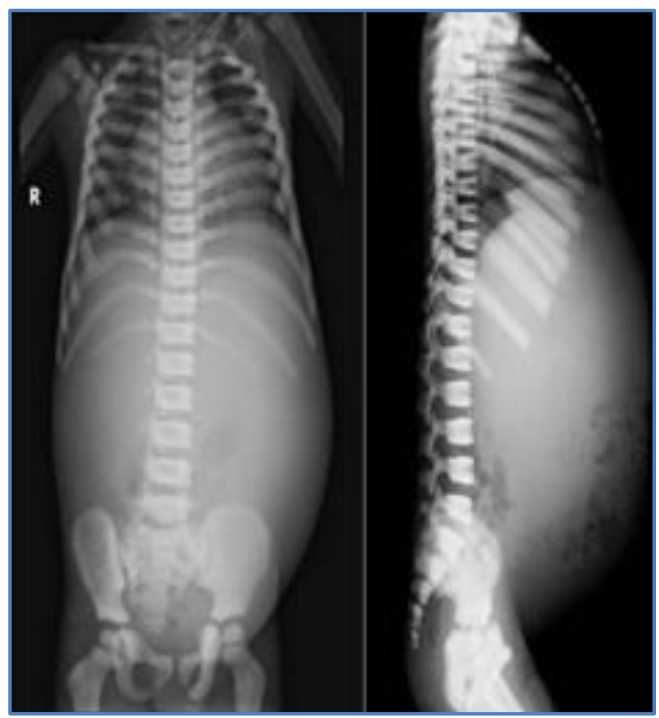

Figure 2: AP and lateral radiographic views of spine: There is increased bone density of visualized ribs, spine and pelvic bones with endobone (Rugger jersey) appearance

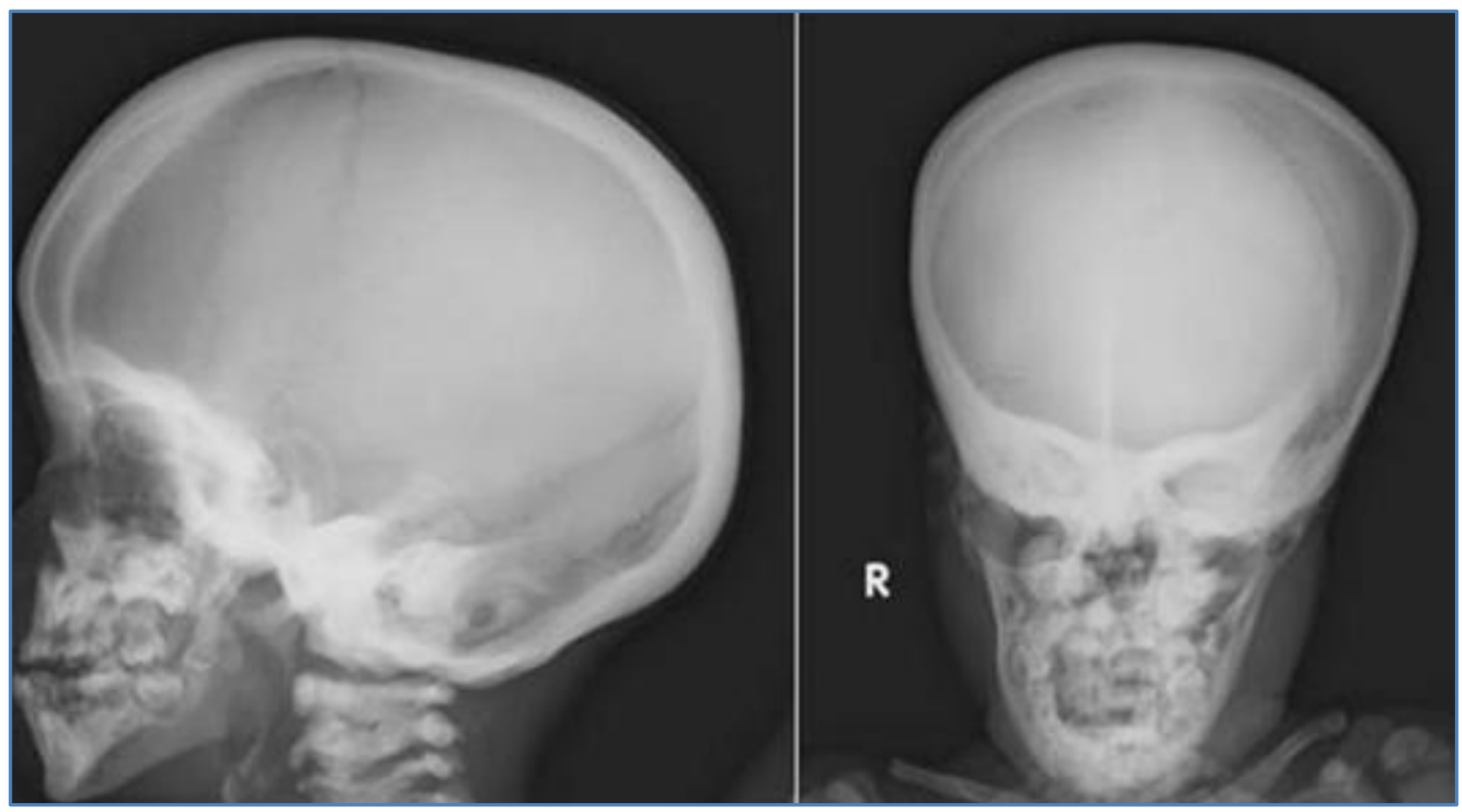

Figure 3: Skull radiogram AP and lateral views: Thickened calvarial bones, base of the skull, orbital ridges and sella turcica

MRI of the head and spine revealed thickened diploic space of the calvarial bones (Fig 4). There is no evidence of optic canal stenosis, dural venous sinuses, and normal posterior fossa (Fig 5). 


\section{CASE REPORT}

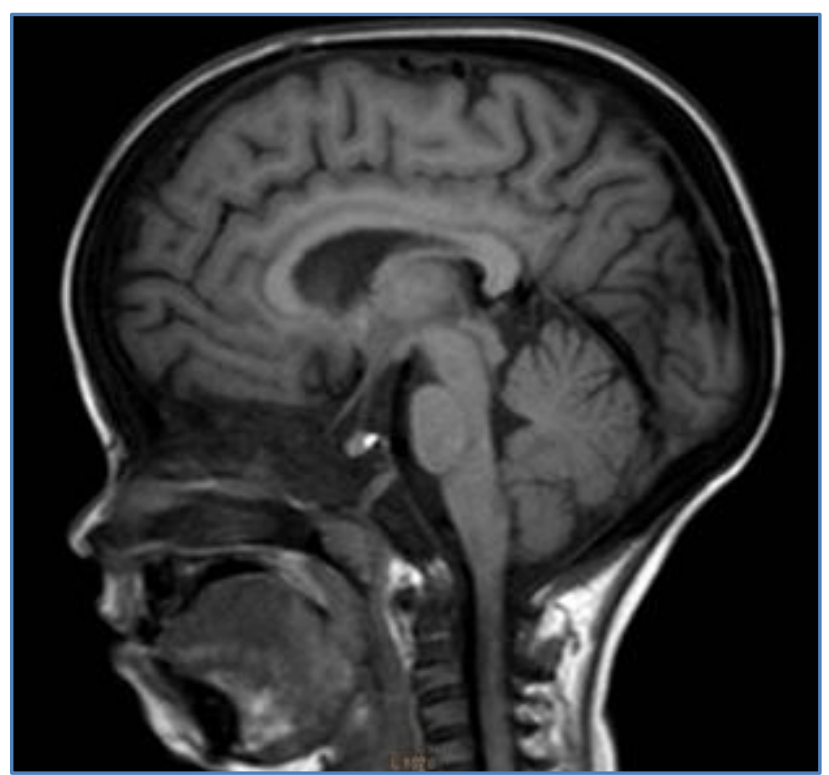

Figure 4: MRI T1W Sagittal image: Thickened calvarial bones significantly thickened base, narrowed pituitary fossa and normal posterior fossa

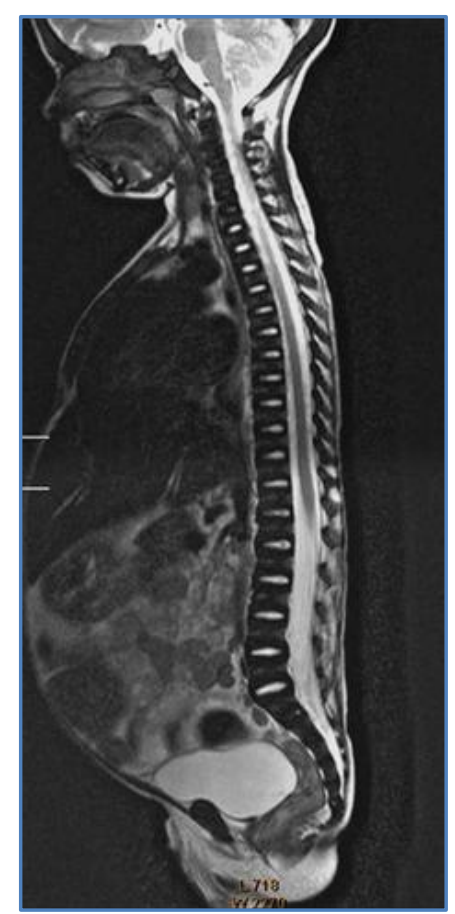

Figure 5: T2W MRI Sagittal image of posterior fossa and spine. Normal posterior fossa and spinal cord

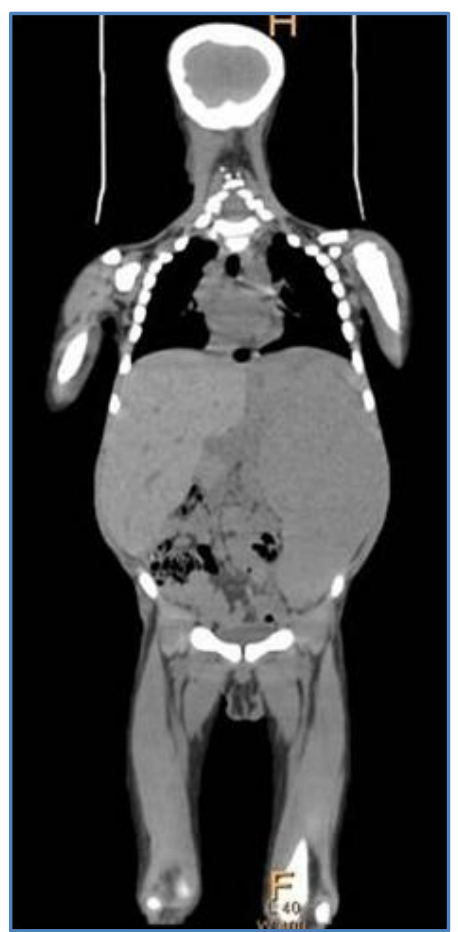

Figure 6: Plain CT reformatted coronal image of the head, thorax and abdomen Thickened calvarial bones and hepatosplenomegaly

Plain CT and ultrasound abdomen showed hepatosplenomegaly with no other abnormality (Fig. 6). 
Based on the clinical presentation, hematological picture and classical radiological features diagnosis of intermediate osteopetrosis was given.

DISCUSSION: Osteopoetrosis is a group of rare congenital dysplastic bone disorder characterized by abnormal osteoclastic function with an incidence of 1:100, 000. About nine forms are identified with different modes of inheritance.

Grossly the disease is classified into severe (autosomal recessive ARO), intermediate (autosomal dominant or recessive) and mild (autosomal dominant) forms. ${ }^{1,4}$ The intermediate osteopetrosis is rare form of OP which manifest in late infancy or after first decade. It is milder form of autosomal recessive condition.

The various manifestations are result of hyperostosis which is due to abnormal osteoclastic function causing defective bone resorption and remodeling. These patients presents with abnormal stature, macrocephaly, anemia, splenomegaly and abdominal distension.

The hematological findings which are seen in the present case are due to obliteration of the bone marrow cavity by the abnormal bone leading to pancytopenia, thrombocytopenia, leukopenia and hemolytic anemia. Hypersplenism and hepatosplenomegaly is due to extramedullary haematopoiesis.1, 3, 4

The radiologic findings are increase in bone density, metaphyseal lines and metaphyseal modeling deformities. The bone within bone appearance is characteristic and diagnostic which is due to the presence of formation of new abnormal bone within the normal outline of the current shadow which is due to the cyclical nature of the disease.1,4,5 Irregular condensation of the bone at metaphysis produces parallel plates of dense bone at the ends of long bone which are present in this case. These patients usually present with hypocalcaemia.2, 4

In our case serum calcium levels were within lower limits of normal. In case series reported by Phadke et al, serum calcium levels were normal. Recurrent infections in patients with osteopetrosis were due to defect in macrophage function. Chronic anemia, recurrent infections, cranial nerve involvements are the causes of failure to thrive in these children.

The definitive treatment of OP is bone marrow transplantation with supportive treatment of anemia and infections.

The importance of radiological investigations is very evident in this case. The plain radiographic findings are crucial in diagnosing OP. However, MRI cranium helps to identify the intracranial complications, the severity of the condition and possibility of reaching the variant of OP. Early recognition and treatment is important to prevent complication and recurrent infections.

CONCLUSION: Osteopetrosis is a relatively a rare congenital condition. The imaging features in addition to history and clinical features are very crucial for diagnosis. Multimodality approach by using plain radiography, multiplanar CT and MRI plays a very important role in establishing the diagnosis.

Although the $\mathrm{X}$ ray findings are very classical in establishing the diagnosis, multiplanar CT and MRI helps in assessing the osseous extent, especially foraminal narrowing in the base of skull and cranial nerve involvement. 


\section{REFERENCES:}

1. Anderson PE, Bollerslev J. Heterogeneity of autosomal dominant Osteopetrosis. Radiology 1987; 164:223-5.

2. Del Fattore A, Cappariello A, Teti A. Genetics, pathogenesis and complications of osteopetrosis. Bone 2008; 42:19-29.

3. Imanimoghaddam M, Davachi B, Nemati S, Johari M, D et al. Radiographic Findings of Malignant Osteopetrosis: Re-port of Four cases. Iran J Radiol 2009; 6:141-5

4. Phadke SR, Gupta A, Pahi J, Pandey A, Gautam P, Agarwal SS. Malignant recessive osteopetrosis. Indian Paediatr 1999; 36:69-74.

5. Wilms G, Casaer P, Alliet P, et al. Cerebrovascular occlusive complications inosteopetrosis major. Neuroradiology1990; 32:511-513.

6. Cure' JK, Key LL, Shankar L, Gross AJ. Petrous carotid canal stenosis in malignant osteopetrosis: CT documentation with MR angiographic correlation. Radiology 1996; 199:415-421.

7. Kahler SG, Burns JA, Ayslworth AS. A mild autosomal recessive form of osteopetrosis. Am J Med Gen 1984; 17:451-464.

8. Joel K. Cure, Lyndon L. Key, David D. Goltra, and Pamela Van Tassel. Cranial MR Imaging of Osteopetrosis.AJNR2000; 21:1110-1115.

9. 00_OP_Guidelines_02122011- - Osteopetrosis - Consensus Guidelines of the ESID and the EBMT Working Party Inborn Errors.

\section{AUTHORS:}

1. Rohini A.

2. Suresh A.

3. Prachi Kala

4. Bala Thimmaiah A.

5. Ramprakash H.V.

\section{PARTICULARS OF CONTRIBUTORS:}

1. Assistant Professor, Department Radiodiagnosis and Imaging, Vydehi Institute of Medical Sciences and Research Centre, (VIMS \& RC), Whitefield, Bangalore.

2. Assistant Professor, Department Radiodiagnosis and Imaging, Vydehi Institute of Medical Sciences and Research Centre, (VIMS \& RC), Whitefield, Bangalore.

3. Professor, Department Radiodiagnosis and Imaging, Vydehi Institute of Medical Sciences and Research Centre, (VIMS \& RC), Whitefield, Bangalore.
4. Professor, Department Radiodiagnosis and Imaging, Vydehi Institute of Medical Sciences and Research Centre, (VIMS \& RC), Whitefield, Bangalore.

5. Professor and HOD, Department Radiodiagnosis and Imaging, Vydehi Institute of Medical Sciences and Research Centre, (VIMS \& RC), Whitefield, Bangalore.

\section{NAME ADDRESS EMAIL ID OF THE CORRESPONDING AUTHOR:}

Dr. Suresh A,

Department of Radiodiagnosis,

VIMS \& RC,

\#82, EPIP Area, Whitefield,

Bangalore - 560066 .

E-mail: majorasuresh@gmail.com

Date of Submission: 07/03/2014.

Date of Peer Review: 08/03/2014.

Date of Acceptance: 23/03/2014.

Date of Publishing: 05/04/2014. 